\title{
Using modified moments for numerical solution of oscillatory integrals
}

\author{
M. Shahbaznezhad, H. Derili* \\ Department of Mathematics, Karaj Branch, Islamic Azad University, Karaj, Iran \\ *Corresponding author E-mail: derili@kiau.ac.ir
}

Copyright () 2014 M. Shahbaznezhad, H. Derili. This is an open access article distributed under the Creative Commons Attribution License, which permits unrestricted use, distribution, and reproduction in any medium, provided the original work is properly cited.

\begin{abstract}
Numerical methods for strongly oscillatory and singular functions are given in this paper. We present an alternative numerical solution for of oscillatory integrals of the general form $\int_{a}^{b} e^{i \omega g(x)}(f(x) /(x+\tau)) d x$

Where $f(x)$ and $g(x)$ are smooth functions in the interval [a,b]. Also $\omega \epsilon R$ and $a<\tau<b$. In order to achieve this goal and avoid singularity, the mentioned integral is solved by the modified moments using interpolating at the Chebyshev points. Finally, we give some experiments for showing efficiency and validity of the method.
\end{abstract}

Keywords: Approximation, Highly Oscillatory Integrals, Hermite Interpolation, Orthogonal Polynomials, Moments, Uniform Approximation.

\section{Introduction}

Integrals of strongly oscillatory or singular functions appear in many branches of mathematics, physics and other applied computational sciences. The standard methods of numerical integration often require too much computation work and cannot be successfully applied. In the fields of electromagnetics, optics, and quantum mechanics, it is very demanding to calculate highly oscillatory integrals rapidly and accurately $[1,2,4,14]$. In this paper, we evaluate the Cauchy principal value integrals including oscillatory functions of the form below numerically:

$I_{\omega}(f, \tau)=\int_{a}^{b} e^{i \omega g(x)} \frac{f(x)}{x+\tau} d x=\lim _{\varepsilon \rightarrow 0^{+}} \int_{|x+\tau| \geq \varepsilon} e^{i \omega g(x)} \frac{f(x)}{x+\tau} d x, a<\tau<b, i^{2}=-1$

Where $f(x)$ has one simple pole in the interval [a,b] and $\omega \in R$ may be large. This equation is applicable in a wide range of practical problems and applications ranging from nonlinear optics to fluid dynamics, plasma transport, computerized tomography, and celestial mechanics.

The integral equation (1) exists where function of $f(x)$ satisfies Hölder's condition on the interval [a, b]. In the integral equation (1) we encounter with two practical difficulties, the first one is it being oscillatory and the second one, having a singularity of Cauchy type [2], [6].

In Okecha [6,7], quadrature rules of interpolatory type is proposed to compute equation (1) numerically where the problem is solved by replacing the function $f(x)$ with the corresponding Lagrange interpolating polynomial on the zeros of the Legendre polynomial and the point $\tau$. Recently, Wang and Xiang have proposed a numerically stable algorithm. Their proposition is based on interpolating $f(x)$ at practical Chebyshev points and avoiding the singularity of the problem [9]. Capobianco and Criscuolo proposed a numerical method based on an interpolatory procedure at the zeros of the orthogonal polynomials with respect to a Jacobi weight [2].

It is a known fact that the interpolation polynomial with the Chebyshev points of the first kind or second kind can lead to the absence of Runge phenomenon.

$(f(x)+f(\tau)) /(x+\tau)$ Is a smooth function for $x \epsilon[a, b]$, then in this paper we propose another approach based on a special approximation of $(f(x)+f(\tau)) /(x+\tau)$ by a truncated series of Chebyshev polynomials as follows

$\frac{f(x)+f(\tau)}{x+\tau}=\sum_{k=0}^{N} a_{k} T_{k}(x)$

The given function satisfies Hermite interpolation's conditions and $T_{k}(x)$ is defined as the Chebyshev polynomial of the first kind by $T_{j}(\cos \theta)=\cos j \theta$. The integral equation (1) is now approximated by 
$I_{\omega}(f, \tau) \simeq \sum_{k=0}^{N} a_{k} M_{k}(\omega)+f(\tau) \int_{a}^{b} \frac{1}{x+\tau} e^{i \omega g(x)} d x$

Where

$M_{k}(\omega)=\int_{a}^{b} T_{k}(x) e^{i \omega x} d x$

Are called modified moments.

The paper is organized as follows. In Section 2, we present the details of the proposed method for computing the Moments. In Section 3, we give an error analysis. Finally, In Section 4 we show that the proposed method is effective by some numerical examples.

\section{Description of method}

The integral equation (1) is rewritten:

$I_{\omega}(f, \tau)=I_{\omega}(f(x)+f(\tau), \tau)-f(\tau) I_{\omega}(1, \tau)$,

Where

$I_{\omega}(f(x)+f(\tau), \tau)=\int_{a}^{b} e^{i \omega g(x)}((f(x)+f(\tau)) /(x+\tau)) d x$

And

$I_{\omega}(1, \tau)=\int_{a}^{b} e^{i \omega g(x)}(1 /(x+\tau)) d x$

Function $f(x)$ is a smooth function in [a, b].

From $[2,6,7,9]$, the integral $I_{\omega}(1, \tau)$ can be calculated by the following analytical results:

$\operatorname{Re}\left[I_{\omega}(1, \tau)\right]=\cos (\tau \omega)\left[\operatorname{Ci}\left(u_{1}\right)-\operatorname{Ci}\left(\left|u_{2}\right|\right)\right]-\sin (\tau \omega)\left[\operatorname{Si}\left(u_{1}\right)+\operatorname{Si}\left(\left|u_{2}\right|\right)\right]$

And

$\operatorname{Im}\left[I_{\omega}(1, \tau)\right]=\sin (\tau \omega)\left[\operatorname{Ci}\left(u_{1}\right)-\operatorname{Ci}\left(\left|u_{2}\right|\right)\right]+\cos (\tau \omega)\left[\operatorname{Si}\left(u_{1}\right)+\operatorname{Si}(|u 2|)\right]$

Where $u_{1}=\omega(1-\tau), u_{2}=-\omega(1+\tau), \mathrm{Ci}$ and $\mathrm{Si}$ are cosine and sine integrals, defined respectively as follows

$C i(u)=\int_{0}^{u} \frac{\cos (x)-1}{x} d x+\log (u)+C=\sum_{n=1}^{\infty} \frac{(-1)^{n} u^{2 n}}{2 n(2 n) !}+\log (u)+C$

$\operatorname{Si}(u)=\int_{0}^{u} \frac{\sin (x)}{x} d x=\sum_{n=0}^{\infty} \frac{(-1)^{n} u^{2 n+1}}{(2 n+1)(2 n+1) !}$

Where $C$ is the Euler constant [1].

The main discussion of this paper is to compute the first integral $I_{\omega}(f(x)-f(\tau), \tau)$ in Equation $(5) . I_{\omega}(f(x)-f(\tau), \tau)$ Is approximated based on the following proposal [4].

Let Us s be a positive integer and $\left.\left\{m_{k}\right\}\right|_{0} ^{N}$ be a set of multiplicities associated with the node points $a=C_{0}<C_{1}<\cdots<$ $C_{N}=b$ where $m_{0}, m_{N} \geq s$. Suppose that $v(x)=\sum_{k=0}^{n} a_{k} T_{k}(x)$, (where $n=\sum_{k=0}^{n} m_{k}-1$ and $T_{k}(x)$ denotes the Chebyshev polynomial of the first kind defined by $\left.T_{k}(\cos \theta)=\cos k \theta\right)$, is the solution of the system of following equations

$v\left(C_{k}\right)=\frac{f\left(C_{k}\right)+f(\tau)}{x+\tau}, \quad v^{\prime}\left(C_{k}\right)=\left[\frac{f\left(C_{k}\right)+f(\tau)}{x+\tau}\right]^{\prime}, \ldots, v^{\left(m_{k}-1\right)}\left(C_{k}\right)=\left[\frac{f\left(C_{k}\right)+f(\tau)}{x+\tau}\right]^{\left(m_{k-1}\right)}$

Where for every integer $0 \leq k \leq N, f^{\left(m_{k}-1\right)}\left(C_{k}\right)$ is the $\left(m_{k-1}\right)$ Th derivative of $f$ at the Chebyshev points or ClenshawCurtis points $C_{k}=\cos (k \pi / N)$.

Remark 1: If some interpolating point $C_{k}=\tau$ exists, then so as to obtain the values of all derivatives of the function $(f(x)+f(\tau))(x+\tau)$ at the point $\tau$ we have:

$V\left(C_{k}\right)=\lim _{x \rightarrow \tau} \frac{f(x)+f(\tau)}{x+\tau}, \quad v^{\prime}\left(C_{k}\right)=\lim _{x \rightarrow \tau}\left[\frac{f(x)+f(\tau)}{x+\tau}\right]^{\prime}, \ldots, v^{\left(m_{k}-1\right)}\left(C_{k}\right)=\lim _{x \rightarrow \tau}\left[\frac{f(x)+f(\tau)}{x+\tau}\right]^{\left(m_{k}-1\right)}$

For $\forall x \in[a, b]$ and $a<\tau<b$ we have,

$I_{\omega}(f, \tau) \simeq I_{(\omega, n)}(f, \tau)=I_{\omega}(v(x))-f(\tau) I_{\omega}(1, \tau),=\sum_{k=0}^{n} a_{k} M_{k}(\omega)+f(\tau) I_{\omega}(1, \tau)$

Where $M_{k}(\omega)=\int_{a}^{b} e^{i \omega x} T_{k}(x) d x$ is the modified moment.

Here, we apply an alternative method to compute the modified moments $M_{k}(\omega)$. Following Abramowitz and Stegun [3], it establishes

$\left(1-x^{2}\right) T_{k}^{\prime}(x)=\frac{k}{2}\left(T_{k-1}(x)-T_{k+1}(x)\right)$,

$2 x T_{k}(x)=T_{k+1}(x)+T_{k-1}(x)$

Therefore,

$-2 x T_{k}(x)+\left(1-x^{2}\right) T^{\prime}{ }_{k}(x)-\left(\frac{k}{2}-1\right) T_{k-1}(x)-\left(\frac{k}{2}+1\right) T_{k+1}(x)$

$K=\int_{-1}^{1}\left(1-x^{2}\right) T_{k}(x) e^{i \omega x} d x$.

Then, using the integration by parts and by Equation (12), we have

$K=\frac{1}{i \omega} \int_{-1}^{1}\left(1-x^{2}\right) T_{k}(x) d e^{i \omega x}$ 
$=\frac{1}{i \omega}\left[\left.\left(1-x^{2}\right) T_{k}(x) e^{i \omega x}\right|_{x=-1} ^{1}-\int_{-1}^{1}\left[\left(1-x^{2}\right) T_{k}(x)\right]^{\prime} e^{i \omega x} d x\right]$

$=-\frac{1}{i \omega} \int_{-1}^{1}\left[-2 x T_{k}(x)+\left(1-x^{2}\right) T^{\prime}{ }_{k}(x)\right] e^{i \omega x} d x$

$=-\frac{1}{i \omega} \int_{-1}^{1}\left[\left(\frac{k}{2}-1\right) T_{k-1}(x)-\left(\frac{k}{2}+1\right) T_{k+1}(x)\right] e^{i \omega x} d x$

$=\frac{1}{i \omega}\left(\frac{k}{2}+1\right) M_{k+1}(\omega)-\frac{1}{i \omega}\left(\frac{k}{2}-1\right) M_{k-1}(\omega)$.

Next, we recalculate $K$ by the second expression of Equation (11), that is,

$K=M_{k}(\omega)-\int_{-1}^{1} x^{2} T_{k}(x) e^{i \omega x} d x$

$=M_{k}(\omega)-\frac{1}{4} \int_{-1}^{1} 2 x\left[T_{k+1}(x)+T_{k-1}(x)\right] e^{i \omega x} d x$

$=M_{k}(\omega)-\frac{1}{4} \int_{-1}^{1}\left[T_{k+2}(x)+T_{k}(x)+T_{k}(x)+T_{k-2}(x)\right] e^{i \omega x} d x$

$=\frac{1}{2} M_{k}(\omega)-\frac{1}{4}\left[M_{k+2}(\omega)+M_{k-2}(\omega)\right]$.

Comparing Equations (14) and (15) yields the recurrence relation

$M_{k+2}(\omega)=-\frac{2(k+2)}{i \omega} M_{k+1}(\omega)+2 M_{k}(\omega)+\frac{2(k-2)}{i \omega} M_{k-1}(\omega)-M_{k-2}$

The two initial moments can be given by

$M_{0}(\omega)=\int_{-1}^{1} T_{0}(x) e^{i \omega x} d x=\frac{2 \sin (\omega)}{\omega}$,

$M_{1}(\omega)=\int_{-1}^{1} T_{1}(x) e^{i \omega x} d x=2\left[\frac{\sin (\omega)}{\omega^{2}}-\frac{\cos (\omega)}{\omega}\right] i$

We know that $T_{-k}(x)=T_{k}(x), k=1,2,3, \ldots$ and consequently $M_{-k}(\omega)=M_{k}(\omega)$.

Substituting $k=0$ and 1 into Equation (16) yields $M_{2}(\omega)$ and $M_{3}(\omega)$, i.e.

$M_{2}(\omega)=-\frac{4}{i \omega} M_{1}(\omega)+M_{0}(\omega)$,

$M_{3}(\omega)=\left[\frac{24}{(i \omega)^{2}}+1\right] M_{1}(\omega)-\frac{8}{i \omega} M_{0}(\omega)$

$M_{k}(\omega) k=4,5 \ldots$ Computed by forward recursion.

If $k \rightarrow \infty$, we have

$M_{k+2}(\omega)+\frac{2 k}{i \omega} M_{k+1}(\omega)-2 M_{k}(\omega)-\frac{2 k}{i \omega} M_{k-1}(\omega)+M_{k-2}(\omega)=0$.

The four characteristic roots of the difference Equation (19) are located inside the unit circle when

$k<\omega$. Hence we deduce that the forward recursion (19) is stable provided $k<\omega$, and for $k \geq \omega$

The forward recursion is no longer applicable. It illustrates that the forward recursion (16) is stable provided $k<\omega$ too.

Remark 2: In all above integrals we transformed the interval [a,b] into [-1, 1]. For this, we used the following transformation for $[a, b]$ :

$t=\frac{b-a}{2} x+\frac{b+a}{2} . x \in[-1,1]$

\section{Error analysis}

In this section, we show that the present rule is of uniform convergence without any restrictions on the location of $\tau \in(a, b)$. To start with the error analysis of the quadrature rule (10) based on Hermite interpolation condition. we first provide the following classical results [10]:

Let $\mathrm{s}$ be some positive integer and let $\left.\left\{m_{k}\right\}\right|_{0} ^{N}$ be a set of multiplicities associated with the node points

$a=c_{0}<c_{1}<\cdots<c_{N}=b$ Such that $m_{0}, m_{N} \geq s$. Suppose that $\phi(x) \in C^{\infty}[a, b]$ satisfies

$\phi\left(C_{k}\right)=\phi^{\prime}\left(C_{k}\right)=\cdots=\phi^{\left(m_{k}-1\right)}\left(C_{k}\right)=0, \quad k=0,1, \ldots, N$

Then $\varphi(x)$ can be represented in the form

$\phi(x)=\frac{\phi^{(n+1)}(\xi)}{(n+1) !} \prod_{k=0}^{N}\left(x-c_{k}\right)^{m_{k}}, \quad x \in[a, b]$

For some $\xi \in[a, b]$ and $n=\sum_{k=0}^{N} m_{k}-1$.

Theorem 3.1: Let $f$ be a smooth function and $\tau \in(a, b)$, then the error of the approximation (10) satisfies the following result

$E_{N+1}(\tau) \leq \frac{2^{n-s+2}(n-s+2)\left\|\phi^{(n+1)}(x)\right\| \infty}{\omega^{s+1}(n-s+1) !}$

Proof: [16].

In particular, when $\mathrm{m}_{\mathrm{k}}=1$, there exists $\mathrm{n}=\mathrm{N}$ and $\mathrm{s}=1$. From Equation (22), we have

$E_{N+1}(\tau) \leq \frac{2^{N+1}(N+1)\left\|\phi^{(N+1)}(x)\right\| \infty}{\omega^{2} N !}$

We can conclude that the error of the quadrature rule (10) tends to zero uniformly as $N \rightarrow \infty$ or the parameter $\omega \rightarrow \infty$. 


\section{Numerical experiments}

The method proposed in this paper is based on the Chebysheves polynomials. So the numerical experiments below will focus on comparing the performance between this method with another method such as Wang and Xiang's method and Okecha's method.

All computations have been performed by using Maple 14 with 64-digit arithmetic.

Experiment 1: Calculate the following oscillatory integral:

$I=\int_{0}^{1} e^{i 200\left(x^{2}+x\right)}\left(e^{10 x} /(x+0.1)\right) d x$

This is similar to an example in [6]. The exact integral value, obtained by the symbolic integral tools with 15 significant digits reserved, is $I=0.00942790128900773+\mathrm{i} \times 0.0977885216048126$.

In this example, we compute the integral by using Equation (10) and compare the present rule (10) with the formula proposed by Okecha [15] and the rule proposed by Wang and Xiang [12,13].

Table 1: Comparison of the present method (10), where $m_{0}=m_{N}=0$ and $m_{1}=\cdots=m_{N-1}=1$, with Okecha's method [15] and Wang and Xiang's method [12,13] for experiment 1 with $\tau=0.1$ and $\omega=200$.

\begin{tabular}{llll}
$\mathrm{N}$ & Present method & Wang and Xiang's method & Okecha's method \\
\hline 2 & $3.91 \times 10^{-8}$ & $5.32 \times 10^{-6}$ & $3.23 \times 10^{-5}$ \\
4 & $5.73 \times 10^{-10}$ & $4.05 \times 10^{-7}$ & $7.19 \times 10^{-5}$ \\
8 & $5.33 \times 10^{-15}$ & $1.20 \times 10^{-14}$ & $6.73 \times 10^{-10}$ \\
16 & $3.26 \times 10^{-26}$ & $9.01 \times 10^{-22}$ & $8,23 \times 10^{-20}$ \\
\hline
\end{tabular}

Experiment 2: Calculate the following oscillatory integral:

$I=\int_{0}^{1} e^{i 100 \tan x}\left(\sec x \cdot e^{-\tan x} /(x+0.1)\right) d x$

This is similar to an example in [17]. The exact integral value is $I=-28.6384665450831+\mathrm{i} \times 17.1789068416939$.

In this example, we compute the integral by using Equation (10) and compare the present rule (10) with the formula proposed by Okecha [15] and the rule proposed by Wang and Xiang [12,13]

Table 2: Comparison of the present method (10), where $m_{0}=m_{N}=0$ and $m_{1}=\cdots=m_{N-1}=1$, with Okecha's method [15] and Wang and Xiang's method [12,13] for experiment 2 with $\tau=0.1$ and $\omega=100$.

\begin{tabular}{clll}
$\mathrm{N}$ & Present method & Wang and Xiang's method & Okecha's method \\
\hline 2 & $5.02 \times 10^{-8}$ & $3.66 \times 10^{-6}$ & $5.12 \times 10^{-5}$ \\
4 & $4.32 \times 10^{-10}$ & $8.24 \times 10^{-7}$ & $5.98 \times 10^{-6}$ \\
8 & $1.76 \times 10^{-15}$ & $7.58 \times 10^{-12}$ & $3.80 \times 10^{-10}$ \\
16 & $4.81 \times 10^{-21}$ & $2.04 \times 10^{-20}$ & $4.27 \times 10^{-19}$ \\
\hline
\end{tabular}

\section{Conclusion}

In this paper, we have presented and tested a numerically stable interpolator type method for handling (1) based on Chebyshev points and the recurrence relation of the modified moments.

From tables 1-2, we can conclude convergence be fasted when $N \rightarrow \infty$.

\section{Acknowledgements}

This work has been funded and supported by Islamic Azad University-Karaj Branch, and the authors are thankful to it.

\section{References}

[1] G.A. Evans, J.R. Webster, A high order, progressive method for the evaluation of irregular oscillatory integrals, Applied Numerical Mathematics 23 (1997) 205-218.

[2] G.A. Evans, J.R. Webster, A comparison of some methods for the evaluation of highly oscillatory integrals, Journal of Computational and Applied Mathematics 112 (1999) 55-69.

[3] M. Abramowitz and I.A. Stegun, Handbook of Mathematical Functions, National Bureau of Standards, Washington, DC, 1964.

[4] M.R. Capobianco and G. Criscuolo, On quadrature for Cauchy principal value integrals of oscillatory functions, J. Comput. Appl. Math. 156 (2003), pp. 471486

[5] P.I. Davis and P. Rabinowitz, Methods of Numerical Integration, 2nd ed., Academic Press, New York, 1984.

[6] W. Gautschi, Numerical Analysis - An Introduction, Birkhäuser, Basel, 1997.

[7] [I.S. Gradshteyn and I.M. Ryzhik, Tables of Integrals, Series, and Products, $6^{\text {th }}$ ed., Academic Press, San Diego, CA, 2000

[8] L.N.G. Filon, on a quadrature formula for trigonometric integrals, Proc. Roy. Soc. Edinburgh 49 (1928) 38-47.

[9] P.C. Hansen, Rank-Deficient and Discrete Ill-Posed Problems: Numerical Aspects of Linear Inversion, SIAM, Philadelphia, 1998.

[10] D. Huybrechs, on the evaluation of highly oscillatory integrals by analytic continuation, SIAM J. Numer. Anal. 44 (3) (2006) 1026-1048. 
[11] A. Iserles, S.P. Norsett, Efficient quadrature of highly oscillatory integrals using derivatives, Proc. Roy. Soc. 461 (2005) $1383-1399$.

[12] G.E. Okecha, Quadrature formulae for Cauchy principal value integrals of oscillatory kind, Math. Comput. 49 (1987), pp. $259-268$.

[13] G.E. Okecha, Hermite interpolation and a method for evaluating Cauchy principal value integrals of oscillatory kind, Kragujevac J. Math. 29 (2006), pp. 91-98.

[14] J. Oliver, The numerical solution of linear recurrence relations, Numer. Math. 11 (1968), pp. 349-360.

[15] H.Wang and S. Xiang, Uniform approximations to Cauchy principal value integrals of oscillatory functions, Appl. Math. Comput. 215 (2009), pp. $1886-1894$.

[16] S. Xiang, Efficient Filon-type methods for $\int_{a}^{b} e^{i \omega g(x)} f(x) d x$, Numer. Math. 105 (2007), pp. 633-658.

[17] W.C. Chew, Waves and Fields in Inhomogeneous Media, Van Nostrand Reinhold, New York, 1990. 